\title{
Math PhD Careers: New Opportunities Emerging Amidst Crisis
}

\section{Yuliy Baryshnikov, Lee DeVille, and Richard Laugesen}

\begin{abstract}
In light of the shortage of regular academic positions for recent PhDs and the underappreciated opportunities for mathematicians in industry, we describe a model program preparing young mathematicians for a broader range of careers.
\end{abstract}

One of the most troubling trends in the mathematics profession is the shortage of regular positions in academia for recent PhDs, as described in Amy Cohen's op-ed in the October Notices [Coh16].

Our view is that the demand for $\mathrm{PhD}$ mathematicians is there, but we just fail to recognize it. True, many PhD graduates will leave mathematical academia, but that does not mean they must leave mathematics. That mathematics is critically relevant to the needs and challenges of twentyfirst-century society is a cliché, but still true. Scientific and engineering fields have become more quantitative and computational, and the opportunities for mathematicians to contribute wherever sophisticated models are used-be it in big data, neuroscience, climate science, telecommunications-are expanding rapidly. These opportunities are often not explicitly acknowledged by us in our roles as mentors and hence can be hard for our mentees to identify and pursue.

Thus mathematics graduates often pursue such opportunities on their own through a slow and sometimes painful osmosis-like process. Most of us know personally or by sight the students from our departments who have "gone into industry," in many cases after growing weary of the rat race in pursuit of an elusive tenure-track position. Many become quite successful in their new careers (being

Yuliy Baryshnikov is professor of mathematics and electrical and computer engineering at the University of Illinois, UrbanaChampaign. His e-mail address is ymb@ 111 inois s edu.

Lee DeVille is professor of mathematics at the University of Illinois, Urbana-Champaign.

His e-mail address is rdevi11e@i11 inois.edu.

Richard Laugesen is professor of mathematics and director of graduate studies at the University of Illinois, Urbana-Champaign. His e-mail address is 1 augesen@ 111 inois . edu.

For permission to reprint this article, please contact: reprint-permission@ams .org.

DOI: http://dx.doi.org/10.1090/noti1483 smart and industrious people), but few take this route well prepared. Often these graduates fall into their jobs by chance, after some struggle, and frequently with a feeling that an indelible stigma is attached to their nonacademic career choice. This is a sign of our failure as mentors. Regardless of one's opinion on the proper goals of $\mathrm{PhD}$ study, the fact that a significant fraction of graduates from institutions of all types will spend the bulk of their professional lives outside mathematical academia forces

\section{Academia is not a closed ecosystem.} upon us the moral responsibility of providing opportunities that will prepare graduate students for intellectually challeng-

ing and professionally rewarding careers that continue to involve mathematics.

The path from academia to a new career is rarely smooth. Hurdles and challenges-from relatively trivial ones such as learning to code to such existential ones as abandoning the quest for pure knowledge-are abundant along the way. We as advisors rarely have relevant experience ourselves. More often than not, we faculty members are unable to guide our students at this critical juncture in their careers.

We cannot expect to overcome this hurdle by having all mathematical faculty suddenly develop industry connections (although it doesn't hurt to try). But we can choose to speak out in our own departments, in the common rooms, and in faculty meetings, and advising meetings, to say that academia is not a closed ecosystem and that our graduates should explore the full range of options, including the intellectually rewarding, satisfying, and stable jobs outside universities that rely on mathematical expertise and have tangible impacts on our economy and society. Students can seek careers in national labs, in other academic disciplines, in industrial research, in NGOs and governmental research organizations. Mathematics is vital for society to move forward. We just need to start believing our own words and putting them into practice through our students.

Another hurdle faced by students willing to venture "outside" to industry is the reluctance of many potential employers to hire a mathematician. Department heads 


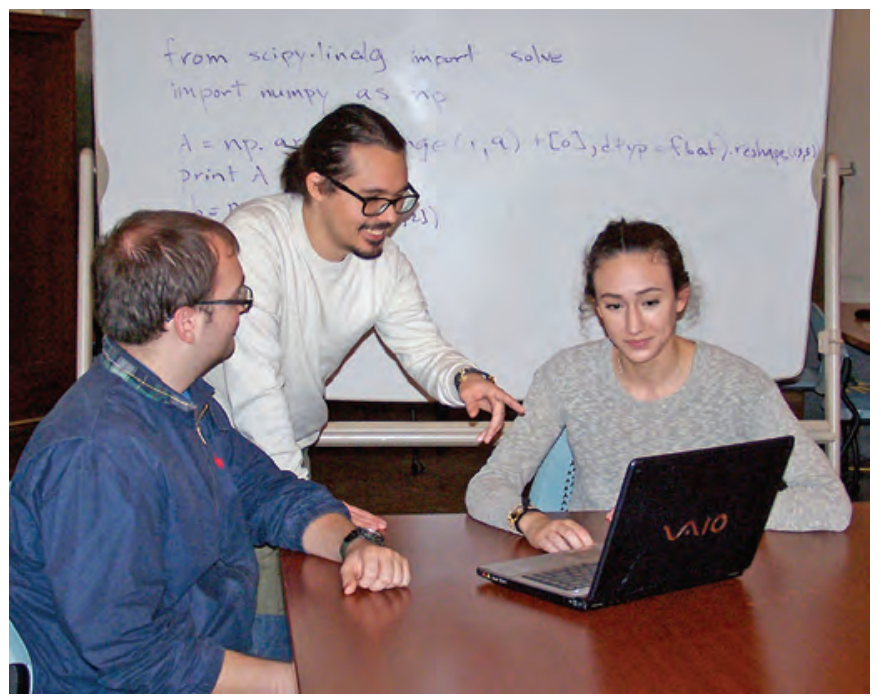

Teaching assistant Stefan Klajbor Goderich (center) helps graduate students William Linz (left) and Colleen Robichaux (right) with a Python programming assignment during the beginning-of-summer Computational Mathematics Bootcamp.

and project managers in industry know what they get by hiring a statistician or an electrical engineer. But a mathematician? What are they good for?! ${ }^{1}$ Unless the enterprise has previously employed $\mathrm{PhD}$ mathematicians, the hiring managers have little experience to draw upon. And indeed, few of our graduates really are ready to walk right in and start work in a typical industrial R\&D environment. The training they need might be minor compared to what they have already learned in graduate school, but still it creates an additional risk and burden that industrial research units might be unwilling to bear.

We do have allies in industry, consisting at a minimum of those of our graduates who went there. A back-ofenvelope calculation ${ }^{2}$ shows that at least six hundred $\mathrm{PhD}$ graduates in mathematics leave academia yearly. The majority of them do not get the word "mathematician" in their job title (indeed, the total number of "mathematicians" outside academia in the US is a paltry three thousand five hundred [USB]). Nonetheless, these individuals work in demanding and technical environments that use their mathematical talent and training at a maximal level. These "hidden mathematicians" enable scientific and technological progress at a level we are generally unaware of. One of the long-term goals for the mathematical community should be to generate broad acceptance

\footnotetext{
${ }^{1}$ V. I. Arnold quoted a famous physicist, Y. Zeldovich, as remarking that formulating a problem precisely is valuable because it allows one to involve mathematicians, who, "like flies, can walk on the ceiling" [Sun04, p. 198]. This attitude is what we would like to see in potential employers!

${ }^{2}$ The number of new PhDs in mathematical sciences is about 1900 per year, and one third of these graduates take their first job in a nonacademic institution. Meanwhile, the number of tenure-track positions under recruitment is fewer than 1000 annually, and not all these positions get filled [AMSa], [AMSb].
}

of the notion of an "in-house" mathematician in every company facing technical or theoretical challenges.

Turning now from diagnosing the problem to proposing solutions, we ask: how can we help our students to overcome career transition hurdles in the short term, and in the longer term create an explicit appreciation in industry and government of the need for mathematically trained personnel?

We believe that at the very least, we need to begin providing to all interested students the skill sets that will enable them to transition more readily to careers outside mathematics departments and that we should educate them, in specific, hands-on ways, about the options outside mathematical departments.

To be clear: we do not advocate turning mathematical graduate education into a training funnel for corporate $R \& D$ or pushing any of our students away from theoretical mathematics or away from academia. However, we should work to change the general level of ignorance among academic mathematicians of what mathematicians can do outside universities and to change the ignorance prevalent in industry of the benefits of hiring mathematicians. These changes can together unlock a huge untapped reservoir of career opportunities for our graduate students.

The good news is that to instigate these changes, we need not radically reshape what our students do during graduate school or how we operate professionally as academics. Small but sustained tweaks in graduate training and a robust effort to connect with potential employers will suffice.

With these principles in mind, at the University of Illinois we created the Program for Interdisciplinary and Industrial Internships at Illinois (PI4), with muchappreciated support from the National Science Foundation under grant DMS MCTP 1345032.

The main goal of the PI4 program is to expose mathematics $\mathrm{PhD}$ students to alternative career paths early in their graduate careers so that they understand what skill sets they should acquire by the time they graduate. Interested students can then prepare for a career path in industry or government during the middle of their $\mathrm{PhD}$ program (rather than in a rush at the end), for example, by picking up coding skills from online MOOCs over winter break or by taking a course in machine learning from the statistics department and also doing one or more internships to develop the soft skills of teamwork and project management. The secondary goal of PI4 is to develop a cadre of supportive local employers who 
have experienced first-hand the value provided to their organization by hiring a mathematics student.

Program components include: in-semester topics courses such as Top Ten Algorithms for the Twenty-First Century, a beginning-of-summer Computational Bootcamp, summer working groups for junior students on exploratory topics such as "Topologically constrained problems of statistical physics, and internship placements for both junior and senior students.

The two-week intensive Computational Bootcamp kicks off the summer, teaching practical techniques with Python programming to students having little or no programming experience. Each day, students:

(i) learn new concepts,

(ii) test their understanding on short programming exercises,

(iii) present and critique student project solutions, and

(iv) code the next project.

The examples typically center around numerical linear algebra, optimization, graph theory algorithms, differential equations, and data fitting. It is an intensive program, with most of the students' days spent programming.

Students then split into three tracks for the remainder of the summer: Prepare, Train, and Intern. The Prepare cohort is typically made up of incoming students and students at the end of the first year of graduate school. They work in a group under the supervision of a faculty member (sometimes local, sometimes external), and it is useful to think of this group as an enhanced Research Experience for Undergraduates, an "REU++." The students spend most of their summer learning about a subject and then some time at the end working on questions related to it. This subject usually has a computational tilt, but it is still open-ended research in some mathematical topic, such as random matrix pencils, for example. Members of the Train cohort tend to be more senior students, with one or two years of graduate school experience, who work in small groups on a focused research problem in mathematics, where simulations and experimentation must be combined with theoretical analysis. These parts of the summer program are useful for all mathematics graduate students as they transition from course-takers to researchers.

The Intern stage is the apex of the program. We directly support about twelve students each summer in two tracks: industrial and interdisciplinary internships.

Industrial internships are hosted by enterprises having a local R\&D outlet. The financial support from the NSF grant enables us to achieve two objectives. First, we can work with smaller firms, such as startups or small research groups at companies that are not considered traditional players in research, that would not normally be able to support interns on their own. Second, the financial support reduces the perceived risk to new industrial partners when they hire the unknown quantity of a mathematics intern. By getting a foot in the door, we lay the groundwork for them to hire interns with company funds in future years. Internship examples include image recognition research at the start-up Personify, weed resistance modeling at

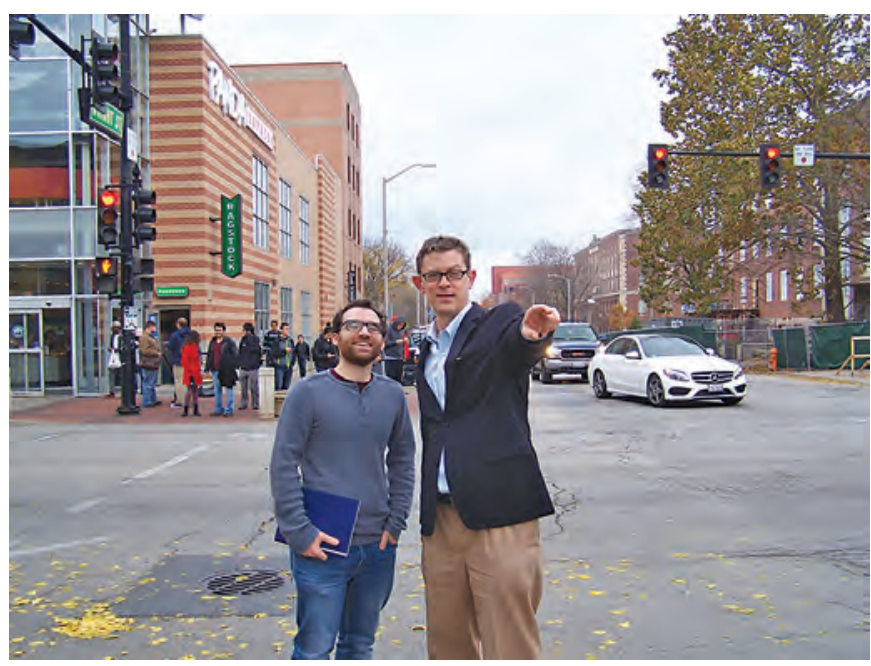

Graduate students Derrek Yager (left) and Vaibhav Karve (not pictured) uncovered low-dimensional structure in New York City taxi data during a scientific internship with Professor Richard Sowers (right). Their work reduced the dimension of the problem from hundreds of thousands down to about fifty dominant roadway combinations.

Dow AgroSciences, and customer data analytics work at utility company Ameren.

Interdisciplinary internships are something completely new for us. We embed a student in a scientific lab on our campus, where they tackle a mathematical problem that supports the agenda of that lab. Almost always the student works in the lab space or is colocated with the group in some other manner. We request that the mentors think of this student as a member of the lab, so that the student learns the techniques required and soaks up the culture of the field. We really want the student to get a feel for what it is like to work in that discipline and how one can contribute with mathematics.

Internships attract What we are doing here addresses a students from all areas of mathematics, not just those in traditionally applied fields. critical need in the Recently, for exammathematical ple, a number theorist modeled ant colony behavior in the entomology department, and a logic student an-

alyzed fMRI data as part of a larger project trying to find physiological imprints of tinnitus.

Once the internship culture is established, students start finding their own positions and funding. Our department has 210 graduate students, including 160 in the $\mathrm{PhD}$ program. Internship numbers have grown from six in 2013 to a total of forty-two interns in 2016, with thirty-one internships taking place over the summer and eleven during the fall or spring semesters. 


\section{Intern Success Story}

Byron Heersink (PhD in number theory expected 2017) did a summer internship through the PI4 program on "A Response Threshold Model for Ant Colonies," a project supervised by faculty members Samuel Beshers (Entomology) and Lee DeVille (Mathematics). Byron developed a computational model of the division of labor in social insect colonies. There is a large literature modeling, calibrating, and checking the individualbased rules insects might use for specialization, but it is difficult to determine how these rules turn into colony-level behaviors. Byron's simulations revealed two surprising effects in the models considered: the degree of specialization is mostly independent of the size of the colony, and the overall colony task behavior depends most strongly on relatively few workers most sensitive to each task and not on the average task sensitivity of the colony.

This project was Byron's first experience of mathematics outside the mathematics department, and his first experience of computational modeling. He subsequently took these skills to an internship at Sandia National Laboratories and then to a second internship at HRL Laboratories, and he is now applying for postgraduation positions in industry as well as academia.

Internships are not slowing down the students' time to degree here at Illinois. Students who aim at an industry or government career after doing an internship develop a sharpened focus that speeds up their academic progress.

The type of industry careers sought by $\mathrm{PhD}$ students at Illinois is shifting. Some still aim at the world of finance, but national labs and data science careers are increasingly valued. The PI4 student cohorts are moving through our $\mathrm{PhD}$ program, and we view the coming diffusion of mathematical talent into the broader world as a highly positive development.

We believe what we are doing here addresses a critical need in the mathematical community: the need to open to our graduates a variety of rewarding, creative, and intellectually challenging mathematical careers. Many graduate students will continue to follow a traditional academic career path, but having the option to choose careers in industry and governmental organizations will benefit all of them.

Mathematics departments must change their culture and (some of) their practices in order to remain relevant in the coming century. Certain departments around the country are already doing so. We hope this article about our experiences at Illinois will foster a conversation in many more mathematics departments about how the community can move forward from the current crisis toward new opportunities for our PhD graduates.

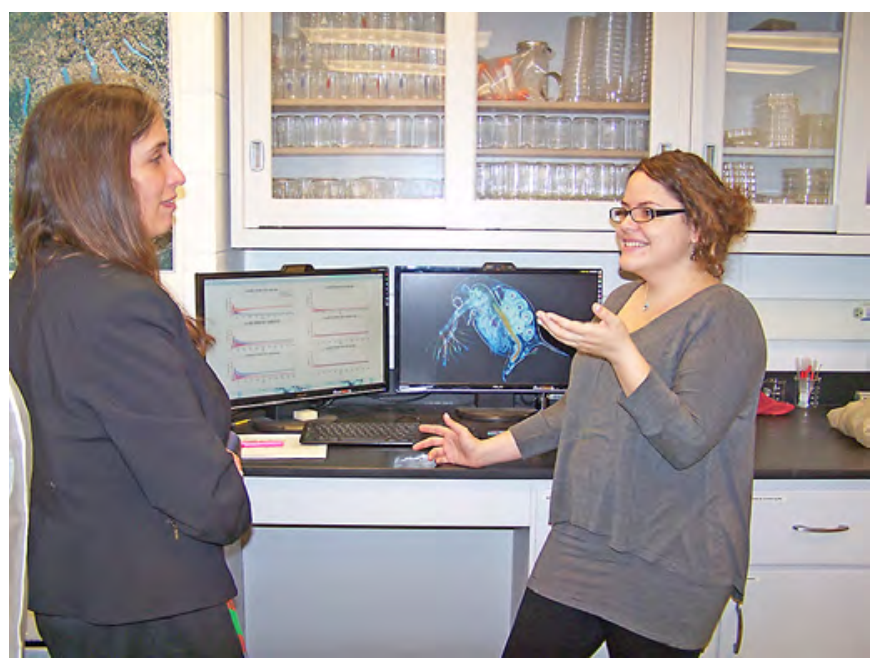

Biology professor Carla Cáceres (left) mentored graduate student Vanessa Rivera Quiñones (right) in her lab for a summer scientific internship. This ongoing work aims to develop biologically realistic SIR population models, applied initially to evolutionary branching in organisms such as water fleas.

\section{National Internship Efforts}

The NSF-IPAM Mathematical Sciences Internship Workshop Report [IPA15] proposes a national effort at multiple scales to provide career and internship placement resources for mathematics graduate students:

- National: create a network to increase internship information exchange, data collection, access, and opportunities;

- Regional: establish internship centers to build internship contacts and organize training opportunities;

- Local: encourage and enable student participation in internships in mathematical sciences departments.

A new BIG Math Network is promoting this concept $(\mathrm{BIG}=$ Business + Industry + Government $)$, which has generated enthusiasm at national societies including AMS, SIAM, MAA, ASA, AWM, NAM, AMATYC, INFORMS, and SACNAS. Supporters who want to volunteer time and effort or who simply want to stay in the loop can keep informed at the website [BIG] and sign up at tinyur 1.com/BIGmathnetwork.

\section{References}

[AMSa] AMS Annual Survey of the Mathematical Sciences: Academic Recruitment and Hiring, www.ams.org/ profession/data/annual-survey/hiring, accessed 2016-10-13.

[AMSb] AMS Annual Survey of the Mathematical Sciences: Doctorates Granted, www. ams . org/profession/data/ annual-survey/docsgrtd, accessed 2016-10-13. 


\section{COMMUNICATION}

[BIG] BIG Math Network,www.siam.org/bigmathnetwork.

[Coh16] AMY CoHEN, Disruptions of the academic math employment market, Notices of the AMS 63 (2016), no. 9, 1057-1060.

[IPA15] NSF-IPAM Mathematical Sciences Internship Workshop Report,goo.g1/QYe3ie, 2015.

[Sun04] RASHID ALIEVICH SunYAev (ed.), Zeldovich: Reminiscences, CRC Press, 2004.

[USB] United States Department of Labor, Bureau of Labor Statistics, Occupational Outlook Handbook, www.b1s. gov/ooh/math/mathematicians.htm, accessed 201610-13.

\section{Photo Credits}

Photos in article are courtesy of University of Illinois at

Urbana-Champaign.

Photo of Lee Deville is courtesy of Brian Stauffer.

Photo of Richard Laugesen is courtesy of Darrell Hoemann.

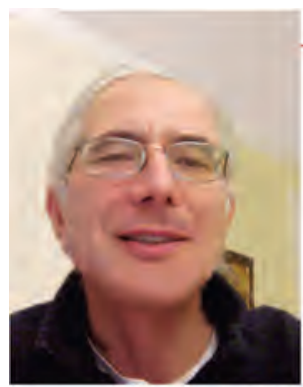

\section{ABOUT THE AUTHORS}

Yuliy Baryshnikov spent a couple of decades in R\&D labs in the fSU and the US and now explores the boiling tedium of the academe.

Yuliy Baryshnikov

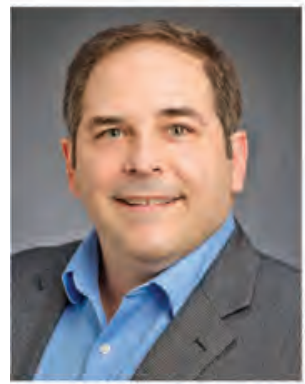

Lee DeVille is an applied(ish) mathematician with interests in dynamical systems, partial differential equations, and stochastic processes. He is greatly interested in finding connections-both in research and in employment-between academic mathematics and that practiced in industry and government.

Lee DeVille

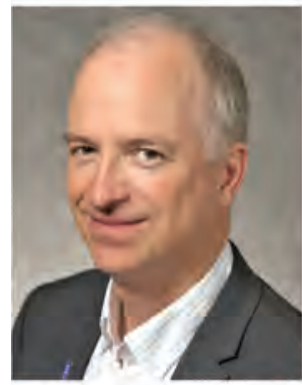

Richard Laugesen enjoys researching and teaching partial differential equations and spectral theory. In addition to promoting mathematical careers in industry and government, he works toward a future in which a lot more women and minority students will pursue graduate-level mathematics.
Richard Laugesen

\section{- I[ЕГП}

Institute for Computational and Experimental Research in Mathematics

\section{SPRING SEMESTER 2018}

\section{Point Configurations in Geometry, Physics and Computer Science}

February 1-May 4, 2018

\section{Organizing Committee:}

Christine Bachoc, University of Bordeaux

Henry Cohn, Microsoft Research - New England

Peter Grabner, Technische Universität Graz

Doug Hardin, Vanderbilt University

Edward Saff, Vanderbilt University

Achill Schürmann, University of Rostock

Sylvia Serfaty, Université Pierre et Marie Curie Paris

Salvatore Torquato, Princeton University

Rob Womersley, University of New South Wales

\section{Program Description:}

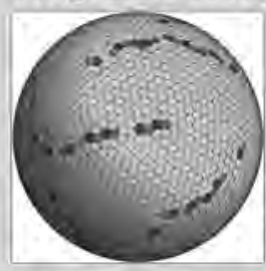
The arrangement of point configurations in metric spaces, whether deterministic or random, is a truly interdisciplinary topic of great interest in mathematics, physics and computer science. Mathematical aspects involve optimization, discretization of manifolds, best packing and cubature, among others. For physics, such configurations arise in the study of crystallization, point processes connected with random matrices, self-assembling materials, jammed states, hyperuniformity and phase transitions. For computer science, extremal point configurations play a fundamental role in coding and information theory, and lattice-based protocols in cryptography and related computational complexity issues are of growing importance. Furthermore, there has been recent and substantial progress on related age-old problems (such as the Kepler conjecture).

Topics for this program include random point configurations, computation and optimization of energy, packing and covering, multi-pole methods, sparsity, and frames, and the theory of lattices with applications to coding and cryptography.

$\begin{array}{ll}\text { Ways to participate: } & \text { About ICERM: The Institute for } \\ \text { Propose a: } & \text { Computational and Experimental } \\ \text { - semester program } & \text { Research in Mathematics is a } \\ \text { - topical workshop } & \text { National Science Foundation } \\ \text { - small group research program } & \text { Mathematics Institute at Brown } \\ \text { - summer undergrad program } & \text { University in Providence, RI. } \\ \text { Apply for a: } & \\ \text { - semester program or } & \\ \text { workshop } & \\ \text { - postdoctoral fellowship } & \text { icerm.brown.edu } \\ \text { Become an: } & \\ \text { - academic or corporate } & \\ \text { sponsor } & \end{array}$

